\title{
Breakfast: to skip or not to skip?
}

\author{
Tanya Zilberter ${ }^{1 *}$ and Eugene Yuri Zilberter ${ }^{2}$ \\ 1 Infotonic Conseil, Marseille, France \\ 2 School of Psychology, University of Glasgow, Glasgow, UK \\ *Correspondence: zilberter@gmail.com \\ Edited by: \\ Maria João Guimarães Fonseca, Centro de Investigação em Biodiversidade e Recursos Genéticos, Portugal \\ Reviewed by: \\ Milka-Dancevic Gojkovic, Public Health Institute of Federation of Bosnia and Herzegovina, Bosnia and Herzegovina
}

Keywords: dietary health education, breakfast skipping, overnight fast, late eating, health risks, intermittent fasting, meal timing, calorie intake

Human eating behaviors are often nonhomeostatic, and thus unlike homeostatic behaviors, they are not exclusively reliant on rigid brain mechanisms, but heavily depend on psychological, sociocultural, and educational factors as well. A clear understanding of the mechanisms and consequences of various eating behaviors is necessary for giving comprehensive educational guidance. However, recommendations regarding breakfast (BF) eating behavior are perhaps the most peremptory yet scientifically (especially metabolically) groundless health guidelines, and thus the widely accepted notion of $\mathrm{BF}$ as the most important meal of the day has been called into question. In a recent meta-analysis, Brown et al. (1), not arguing with the established link between obesity and BF behavior, concluded: "The current body of scientific knowledge indicates that the proposed effect of breakfast on obesity is only presumed true" (p. 1298). The authors state that numerous articles demonstrating negative metabolic effects of skipping BF have yet to establish a causal relationship due to a lack of probative value and that the major obstacle in establishing causality is neglecting the possible confounding factors.

In this opinion paper, we suggest that $\mathrm{BF}$ is just another meal, rather than the "most important meal of the day" as is commonly believed and that prolongation of overnight fast, which depends not only on timing of BF but also on timing of the last meal of the day, can be beneficial.

\section{DEFINITION}

A significant barrier to advances in the study of BF behaviors is the lack of a common language. It is often discussed that there is a fundamental difficulty in comparing different results due to lack of common definitions for both eating $\mathrm{BF}$ and skipping BF [e.g., Ref. $(2,3)]$. Currently, the definition of BF continues to vary between studies, although the definition by Timlin and Pereira (4): "first meal of the day, eaten before or at the start of daily activities (e.g., errands, travel, work), within $2 \mathrm{~h}$ of waking, typically no later than 10:00 in the morning, and of an energy level between 20 and 35\% of total daily energy needs" is accepted as an academic standard. Of significance for this discussion is to note that the above definition does not specify the duration of the overnight fast.

\section{DURATION OF OVERNIGHT FAST}

The duration of overnight fast is rarely specified in studies comparing effects of eating versus skipping BF. As a result, an important parameter, which can significantly influence the metabolic consequences of eating behaviors are neglected in the majority of studies resulting in wrong conclusions (e.g., 4; Figure 1C).

Consider, for example, two BF-eaters having their last meals at midnight; one then consumes BF at 5:00 a.m. (5-h overnight fast) and the other consumes BF at 10:00 a.m. (10-h overnight fast). Both are considered "BF-eaters" in the majority of studies, but the difference in overnight fast durations makes them metabolically distinct from each other. Similarly, "BFeaters" who have an earlier dinner (last meal) at 7:00 p.m. followed by $B F$ at 10:00 a.m. have an overnight fast of $15 \mathrm{~h}$, which can be metabolically similar to "BFskippers" who had a later dinner (midnight) and skipped $\mathrm{BF}$ altogether and ate their first meal at 3:00 p.m. (same 15-h overnight fast). The important point here is that due to the neglect of this important parameter in BF eating behavior studies, it challenges the validity of their findings and the interpretations thereof.

The above point also shines light on potential contradictions in eating behavior literature. For example, although eating late (practicing late dinners and/or nighttime meals) is considered a bad habit and eating $\mathrm{BF}$ is considered healthy, they may both have similar duration of overnight fasts and thus may result in similar metabolic profiles. It is well known that "late eaters" have a problem with losing weight $(5,6)$ and have overall poorer health conditions [Figure 1B, Ref. (7)]. Both late eating and BF skipping are usually listed together as risk factors for unhealthy metabolic profiles [e.g., Ref. $(8,9)]$ but solid evidence exists only for consequences of late eating.

Late or night-time eating was found to be linked to multiple eating behavior pathologies, such as night-time hunger, body image distortions, and mood disorders (10), as well as elevated blood levels of insulin and glucose characteristic for metabolic syndrome (11). Timing of eating behavior has both circadian and noncircadian aspects (12). The non-circadian aspect (that is, 24-h caloric intake) allows for a comparison between the effects of skipping $\mathrm{BF}$ and excluding late eating, in terms of duration of the overnight fast. When late eating is excluded, as it was in a randomized crossover design study (13) where no eating was assigned from 7:00 p.m. to 6:00 a.m. while ad libitum eating was allowed during the rest of the day, the subjects consumed 244 fewer kilocalories regardless of their food choice. The calorie intake factor is one of the most important in determining the metabolic consequences of meals and eating behaviors. 


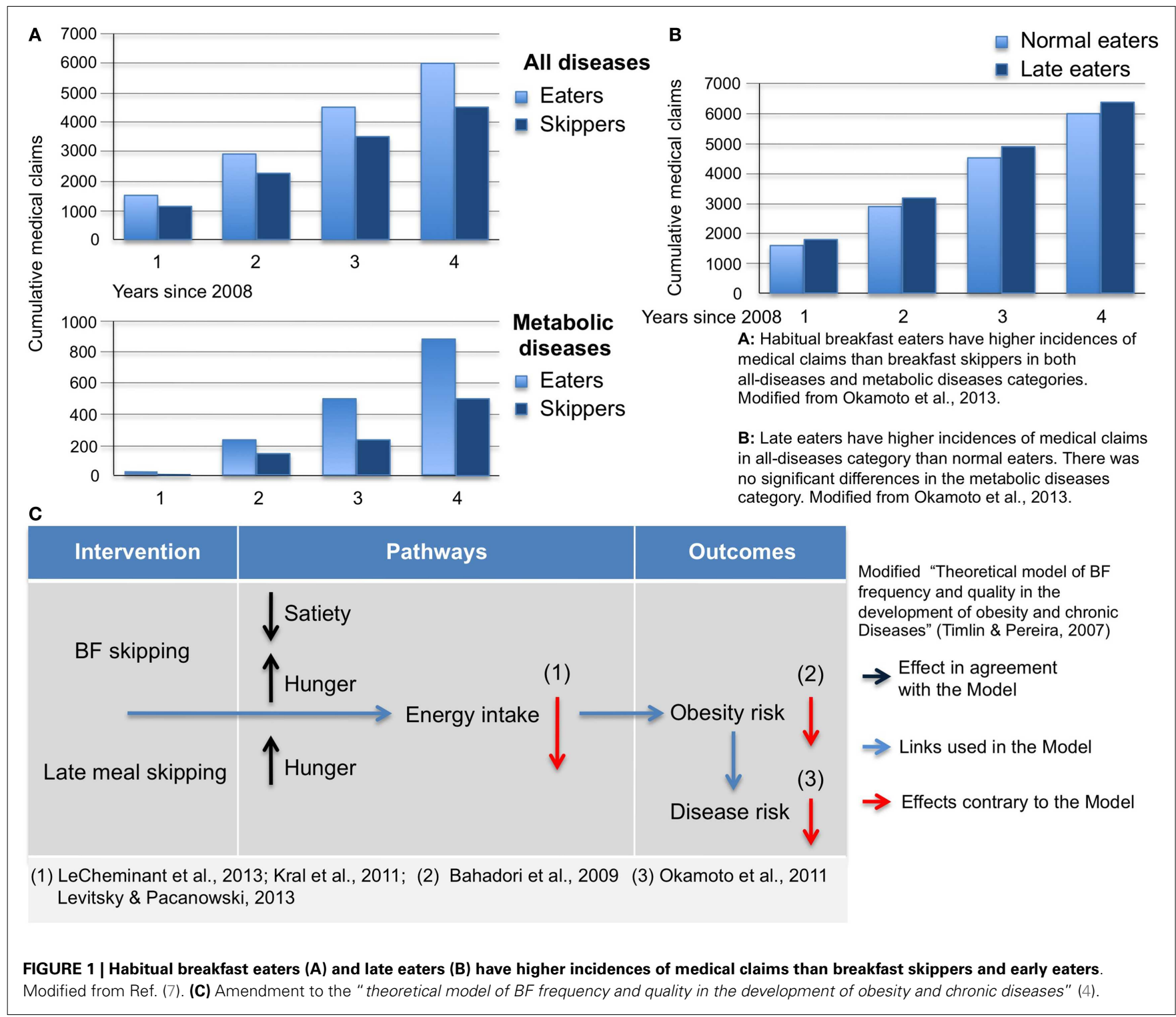

The model proposes that regular BF consumption can lead to increased satiety and decreased hunger [which is in agreement with data of Kral et al. (14) and Levitsky and Pacanowski (15)]. However, research results of Levitsky and Pacanowski (15) do not support this claim. The lower risk of chronic disease suggested in the Model is in disagreement with data depicted in Figures 1A,B.

\section{BREAKFAST SKIPPING: WHAT DO WE REALLY KNOW?}

To investigate the link between BF skipping, BMI, and risks of obesity, a closer look at caloric intake may be used as a tool to establish whether or not there is any causality between them. Calorie intake is considered the major determinant of BMI [e.g., Ref. (16)]. It is routinely stated that skipping BF is overcompensated with increased energy consumption later during the day. Indeed, many authors state that skipping BF results in increased BMIs [e.g., Ref. $(17,18)$ ] although (as mentioned in the introduction) no causality has been established. Additionally, even the very existence of such a link is questioned. While skipping $\mathrm{BF}$ has been linked to higher risk of obesity in the UK (19), Hong Kong (20), and the USA (21), no such link was observed in Australia
(22), Portugal (23), and Saudi Arabia (24).

A link between skipping BF and obesity is constantly being challenged and in many studies, a lack of this link was repeatedly demonstrated (24-32). In fact, the exact opposite link was recently demonstrated: in a large cohort, 4-year long study based on Japanese insurance statistics (7), the accumulation of newly diagnosed diseases was plotted against various lifestyle-related behaviors. Self-reported BF-skippers had a lower incidence of all diseases (including metabolic diseases) as compared to BFeaters. In the same study, a link between late eating and poorer health was demonstrated (Figure 1A). 


\section{CONTROLLED STUDIES OF ENERGY \\ BALANCE}

The supposed disadvantages of skipping BF have not been supported by recent controlled studies. Kral et al. (14) directly demonstrated that skipping BF caused an increase in hunger levels but not in a overcompensation of calorie intake later in the day. On the contrary, Gonzalez et al. (33) found that daily energy and fat intakes were reduced with BF omission. Similarly, Levitsky and Pacanowski (15) showed that, although skipping BF significantly increased hunger ratings at lunchtime, food intake at lunch were not increased. Moreover, skipping BF resulted in a net energy deficit of about $400 \mathrm{kcal}$ a day comparing to BF eating group. Levitsky and Pacanowski mention that 25\% of Americans now regularly skip BF in order to lose weight compared to $14 \%$ in 1965, despite aggressive campaigns labeling skipping $\mathrm{BF}$ as one of the most harmful eating behaviors. The caloric intake reduction due to skipping $\mathrm{BF}$ may offer an explanation for why this practice for successful weight loss works and is on the rise. A similar mechanism of caloric intake reduction due to skipping $\mathrm{BF}$ is present due to abstaining from late eating, as demonstrated by LeCheminant et al. (13).

Another component of energy balance is energy expenditure. Recently, it was shown that skipping BF did not affect 24-h energy expenditure, resting metabolic level, or food-induced thermogenesis (34). This seems to contradict the description of BF-skippers as more sedentary, having decreased energy expenditure [e.g., Ref. (35)]. However, it cannot be excluded that a third factor exists which underlies both skipping BF behavior and sedentary lifestyle (36). In a recent Danish longitudinal study of 8- to 11-year-old children, examining the causality between being overweight and physically inactive, a causality was indeed found, but the exact opposite to what is commonly believed, "that adiposity is a better predictor of physical activity and sedentary behavior changes than the other way around" [(37), p. 1]. If adiposity is, in fact, the reason for physical inactivity, then by the same token, BF skipping may be a result of being overweight, and not the other way around.

\section{INDIRECT EVIDENCE OF THE POTENTIAL BENEFITS OF SKIPPING BREAKFAST}

Comparing to evidence from the broader field of nutrition, we can investigate a potential overlap, which can shine light on the potential benefits of skipping BF. In terms of meal timing, skipping $\mathrm{BF}$ is similar to intermittent fasting (38), however, it is seldom looked at from this point of view. One of metabolic effects of intermittent fasting is intermittent ketosis known for its appetite suppression effect (39-41) resulting in voluntary calorie reduction [e.g., Ref. (42)]. BF skipping and exclusion of late eating, as described above, also result in reduction of voluntary calorie intake $(15,33)$. Calorie restriction has been shown to have profound metabolic benefits including neuroprotective, anti-aging, and anti-inflammatory [see Ref. (43) for review]. Furthermore, Mattson and colleagues showed in rodents that intermittent fasting had more metabolic benefits than permanent calorie restriction (44), thus skipping BF may be more beneficial than traditional restrictive dieting.

\section{CONCLUSION}

Given body of evidence reviewed in this opinion article, it is reasonable to suppose that skipping $\mathrm{BF}$ could be as metabolically beneficial as excluding late eating, as well as stress the importance of the overnight fast. Perhaps it does not matter which of the daily meals - the first or the last - is omitted as long as at least once in a while, an inter-meal interval is long enough to allow the state of ketosis to initiate lipolysis and lower calorie intake, thus decreasing the risk of obesity and its comorbidities.

\section{REFERENCES}

1. Brown AW, Brown MMB, Allison DB. Belief beyond the evidence: using the proposed effect of breakfast on obesity to show 2 practices that distort scientific evidence. Am J Clin Nutr (2013) 98(5):1298-308. doi:10.3945/ajcn.113.064410

2. Rampersaud GC, Pereira MA, Girard BL, Adams J, Metzl JD. Breakfast habits, nutritional status, body weight, and academic performance in children and adolescents. JAm Diet Assoc (2005) 105(5):743-60. doi:10.1016/j.jada.2005.02.007

3. Cuenca-García M, Ruiz JR, Ortega FB, Labayen I, González-Gross M, Moreno LA, et al. Association of breakfast consumption with objectively measured and self-reported physical activity, sedentary time and physical fitness in European adolescents: the HELENA (Healthy Lifestyle in Europe by Nutrition in Adolescence) Study. Public Health Nutr (2013) 105:1-11. doi:10.1017/ S1368980013002437

4. Timlin MT, Pereira MA. Breakfast frequency and quality in the etiology of adult obesity and chronic diseases. Nutr Rev (2007) 65:268-81. doi:10.1111/ j.1753-4887.2007.tb00304.x

5. Cleator J, Abbott J, Judd P, Sutton C, Wilding JPH. Night eating syndrome: implications for severe obesity. Nutr Diabetes (2012) 2(9):e44. doi:10. 1038/nutd.2012.16

6. Baron KG, Reid KJ, Van Horn L, Zee PC. Contribution of evening macronutrient intake to total caloric intake and body mass index. Appetite (2012) 60(1):246-51. doi:10.1016/j.appet.2012.09. 026

7. Okamoto E, Hiratsuka Y, Otsubo K, Kumakawa T. Evaluation of the health check up and guidance program through linkage with health insurance claims. J Natl Inst Public Health (2013) 62(1):13-30.

8. Striegel-Moore RH, Wilson GT, DeBar L, Perrin N, Lynch F, Rosselli F, et al. Cognitive behavioral guided self-help for the treatment of recurrent binge eating. J Consult Clin Psychol (2010) 78(3):312. doi:10.1037/a0018915

9. Cahill LE, Chiuve SE, Mekary RA. Skipping breakfast or eating late at night increases risk of coronary heart disease. Circulation (2013) 128(4):337-43. doi:10.1161/CIRCULATIONAHA.113.001474

10. Boseck JJ, Engel SG, Allison KC, Crosby RD, Mitchell JE, de Zwaan M. The application of ecological momentary assessment to the study of night eating. Int J Eat Disord (2007) 40(3):271-6. doi:10.1002/eat.20359

11. Allison KC, Ahima RS, O'Reardon JP, Dinges DF, Sharma V, Cummings DE, et al. Neuroendocrine profiles associated with energy intake, sleep, and stress in the night eating syndrome. J Clin Endocrinol Metab (2005) 90(11):6214-7. doi:10.1210/jc.2005-1018

12. Gallant AR, Mathieu ME, Lundgren JD, Allison K, Tremblay A, O'Loughlin J, et al. Daily physical activity patterns of children with delayed eating behaviors. J Biol Rhythms (2013) 28(5):332-8. doi:10.1177/0748730413499857

13. LeCheminant JD, Christenson E, Bailey BW, Tucker LA. Restricting night-time eating reduces daily energy intake in healthy young men: a shortterm cross-over study. Br J Nutr (2013) 23:1-6. doi:10.1017/S0007114513001359

14. Kral TV, Whiteford LM, Heo M, Faith MS Effects of eating breakfast compared with skipping breakfast on ratings of appetite and intake at subsequent meals in 8- to 10-year-old children. Am J Clin Nutr (2011) 93:284-91. doi:10.3945/ajcn. 110.000505

15. Levitsky DA, Pacanowski CR. Effect of skipping breakfast on subsequent energy intake. Physiol Behav (2013) 119:9-16. doi:10.1016/j.physbeh. 2013.05.006

16. Bernardin P, Moller A. Recommended daily caloric intake. FASEB J (2013) 27.

17. Van Lippevelde W, Te Velde SJ, Verloigne $M$, Van Stralen MM, De Bourdeaudhuij I, Manios $\mathrm{Y}$, et al. Associations between family-related factors, breakfast consumption and BMI among 10-to 12-year-old European children: the cross-sectional 
ENERGY-study. PLoS One (2013) 8(11):e79550. doi:10.1371/journal.pone.0079550

18. Timlin MT, Pereira MA, Story M, NeumarkSztainer D. Breakfast eating and weight change in a 5 -year prospective analysis of adolescents: Project EAT (eating among teens). Pediatrics (2008) 121(3):e638-45. doi:10.1542/peds.2007-1035

19. Harding S, Teyhan A, Maynard MJ, Cruickshank JK. Ethnic differences in overweight and obesity in early adolescence in the MRC DASH study: the role of adolescent and parental lifestyle. Int J Epidemiol (2008) 37:162-72. doi:10.1093/ije/dym 252

20. So HK, Nelson EA, Li AM, Guldan GS, Yin J, Ng PC, et al. Breakfast frequency inversely associated with BMI and body fatness in Hong Kong Chinese children aged 9-18 years. Br J Nutr (2011) 106:742-51. doi:10.1017/S0007114511000754

21. Laska MN, Murray DM, Lytle LA, Harnack LJ. Longitudinal associations between key dietary behaviors and weight gain over time: transitions through the adolescent years. Obesity (2012) 20:118-25. doi:10.1038/oby.2011.179

22. William P. Breakfast and the diets of Australian children and adolescents: an analysis of data from the 1995 National Nutrition Survey. Int J Food Sci Nutr (2007) 58:201-16. doi:10.1080/ 09637480701198075

23. Mota J, Fidalgo F, Silva R, Ribeiro JC, Santos R, Carvalho J, et al. Relationship between physical activity, obesity and meal frequency in adolescents. Ann Hum Biol (2008) 35:1-10. doi:10.1080/ 03014460701779617

24. Abalkhail B, Shawky S. Prevalence of daily breakfast intake, iron deficiency anaemia and awareness of being anaemic among Saudi school students. Int J Food Sci Nutr (2002) 53:519-28. doi:10.1080/ 09637480220164370

25. Resnicow K. The relationship between breakfast habits and plasma cholesterol levels in schoolchildren. J Sch Health (1991) 61:81-5. doi:10.1111/j. 1746-1561.1991.tb03242.x

26. Sampson AE, Dixit S, Meyers AF, Houser R Jr. The nutritional impact of breakfast consumption on the diets of inner-city African-American elementary school children. J Natl Med Assoc (1995) 87:195-202.

27. Williams P. Breakfast and the diets of Australian adults: an analysis of data from the 1995 national nutrition survey. Int J Food Sci Nutr (2005) 56:65-79. doi:10.1080/09637480500082108

28. Forslund HB, Lindroos AK, Sjostrom L, Lissner L. Meal patterns and obesity in Swedish women - a simple instrument describing usual meal types, frequency and temporal distribution. Eur J Clin Nutr (2002) 56:740-7. doi:10.1038/sj.ejcn.1601387

29. Fujiwara T. Skipping breakfast is associated with dysmenorrhea in young women in Japan. Int J Food Sci Nutr (2003) 54:505-9. doi:10.1080/ 09637480310001622369

30. Nicklas TA, Morales M, Linares A, Yang SJ, Baranowski T, De Moor C, et al. Children's meal patterns have changed over a 21-year period: the Bogalusa Heart Study. J Am Diet Assoc (2004) 104:753-61. doi:10.1016/j.jada.2004.02.030

31. Williams BM, O'Neil CE, Keast DR, Cho S, Nicklas TA. Are breakfast consumption patterns associated with weight status and nutrient adequacy in African-American children? $P u b$ lic Health Nutr (2009) 12:489-96. doi:10.1017/ S1368980008002760

32. Kim JH, So WY. Association between frequency of breakfast eating and obesity in Korean adolescents. Iran J Public Health (2012) 41(6):50-7.

33. Gonzalez JT, Veasey RC, Rumbold PL, Stevenson EJ. Breakfast and exercise contingently affect postprandial metabolism and energy balance in physically active males. Br J Nutr (2013) 110:1-12. doi:10.1017/S0007114512005582

34. Kobayashi F, Ogata H, Omi N, Nagasaka S, Yamaguchi S, Hibi M, et al. Effect of breakfast skipping on diurnal variation of energy metabolism and blood glucose. Obes Res Clin Pract (2013) 8(3):e249-e257. doi:10.1016/j.orcp.2013.01.001

35. Marquez A, Forester S, Witbracht M, Campbell C, Gustafson M, Keim N. Skipping breakfast is associated with lower physical activity energy expenditure in young healthy women. FASEB J (2012) 820:1-11.

36. Odegaard AO, Jacobs DR, Steffen LM, Van Horn L, Ludwig DS, Pereira MA. Breakfast frequency and development of metabolic risk. Diabetes Care (2013) 36:3100-6. doi:10.2337/dc13-0316

37. Hjorth MF, Chaput JP, Ritz C, Dalskov SM, Andersen R, Astrup A, et al. Fatness predicts decreased physical activity and increased sedentary time, but not vice versa: support from a longitudinal study in 8-to 11-year-old children. Int J Obes (2013). doi:10.1038/ijo.2013.229

38. Zilberter T, Zilberter EY. Breakfast and cognition: sixteen effects in nine populations, no single recipe. Front Hum Neurosci (2013) 7:631. doi:10.3389/ fnhum.2013.00631

39. Scharrer E. Control of food intake by fatty acid oxidation and ketogenesis. Nutrition
(1999) 15:704-14. doi:10.1016/S0899-9007(99) 00125-2

40. la Fleur SE, Ji H, Manalo SL, Friedman MI, Dallman MF. The hepatic vagus mediates fatinduced inhibition of diabetic hyperphagia. Diabetes (2003) 52:2321-30. doi:10.2337/diabetes.52. 9.2321

41. Horn CC, Ji H, Friedman MI. Etomoxir, a fatty acid oxidation inhibitor, increases food intake and reduces hepatic energy status in rats. Physiol Behav (2004) 81:157-62. doi:10.1016/j.physbeh. 2004.01.007

42. Dutton SB, Sawyer NT, Kalume F, Jumbo-Lucioni P, Borges K, Catterall WA, et al. Protective effect of the ketogenic diet in Scnla mutant mice. Epilepsia (2011) 52(11):2050-6. doi:10.1111/j.1528-1167. 2011.03211.x

43. Willcox BJ, Willcox DC. Caloric restriction, caloric restriction mimetics, and healthy aging in Okinawa: controversies and clinical implications. Curr Opin Clin Nutr Metab Care (2014) 17(1):51-8. doi:10.1097/MCO.0000000000000019

44. Mattson MP, Duan W, Guo Z. Meal size and frequency affect neuronal plasticity and vulnerability to disease: cellular and molecular mechanisms. J Neurochem (2003) 84:417-31. doi:10. 1046/j.1471-4159.2003.01586.x

Conflict of Interest Statement: The authors declare that the research was conducted in the absence of any commercial or financial relationships that could be construed as a potential conflict of interest.

Received: 30 April 2014; accepted: 16 May 2014; published online: 03 June 2014

Citation: Zilberter T and Zilberter EY (2014) Breakfast: to skip or not to skip? Front. Public Health 2:59. doi: 10.3389/fpubh.2014.00059

This article was submitted to Public Health Education and Promotion, a section of the journal Frontiers in Public Health.

Copyright (c) 2014 Zilberter and Zilberter. This is an open-access article distributed under the terms of the Creative Commons Attribution License (CC BY). The use, distribution or reproduction in other forums is permitted, provided the original author(s) or licensor are credited and that the original publication in this journal is cited, in accordance with accepted academic practice. No use, distribution or reproduction is permitted which does not comply with these terms. 